\title{
History of $\beta$ blockers in congestive heart failure
}

\author{
K Swedberg
}

Twenty years ago, digitalis and diuretics were the mainstay of therapeutic options for patients with idiopathic dilated congestive cardiomyopathy and advanced heart failure. Vasodilator treatment was yet to be established and cardiac transplantation was in its infancy. The historical basis for using $\beta$ blockers to treat heart failure dates back to 1973 when a single bolus injection of practolol was administered to reduce the heart rate of a 59 year old woman with tachycardia caused by an acute pulmonary oedema owing to dilated cardiomyopathy. ${ }^{1}$ The patient was successfully maintained on a twice daily oral regimen of alprenolol, which dramatically improved her heart failure. The patient was later switched to the cardioselective $\beta$ blocker metoprolol. At 82 years old her condition remains stable on metoprolol, enalapril, diuretics, and digitalis.

The clinical outcome of the first seven patients to be treated with $\beta$ blockers were described in $1975 .{ }^{1}$ Six patients received oral practolol 50-400 mg twice daily and one received alprenolol $50 \mathrm{mg}$ twice daily. The duration of treatment ranged from 2-12 months (mean, 5.4 months). All seven patients experienced an improvement in their clinical condition shortly after starting treatment. This was accompanied by haemodynamic evidence of improved ventricular function with progressive improvement in functional capacity and a reduction in heart size.

Based on these preliminary findings a formal, open label study of metoprolol was conducted in 28 patients with heart failure caused by congestive cardiomyopathy, ${ }^{23}$ diagnosed according to the criteria of Goodwin and Oakley. ${ }^{4}$ The patients each completed a one month observation period during which they continued to receive established regimens of diuretics and digitalis. In addition, they received $\beta$ blocker treatment for between two and 62 months (mean, 23 months). Six of the first seven patients received practolol, the next nine received alprenolol (25 or $100 \mathrm{mg}$ bid), and subsequent patients received metoprolol 25-100 mg bid.

Echocardiographic and pulse curve findings indicated that $\beta$ blocker treatment resulted in an improvement in both systolic and diastolic function. ${ }^{3}$ Ejection fraction increased from a mean of $32 \%$ to $42 \%$, and the third heart sound amplitude and rapid filling wave were significantly reduced. Functional classification improved in 15 patients and remained stable in 12 of the remaining 13 patients. Ten patients died but mortality was lower than expected in this severely ill group of patients.
These data provided evidence to suggest that patients with congestive cardiomyopathy may have inappropriate sympathetic cardiac stimulation that can be overcome by chronic $\beta$ blockade. On the basis of these findings it was recommended that $\beta$ blockers be added to conventional treatment regimens (digitalis and diuretics) in all patients with severe heart failure caused by congestive cardiomyopathy.

The prognosis of 24 patients from this study was compared with a that of a retrospectively selected control group of 13 patients who had similar clinical findings and myocardial function (severe congestive cardiomyopathy with a left ventricular end diastolic diameter $>7 \mathrm{~cm}) .{ }^{5}$ All patients were receiving digitalis and diuretics, but the 24 study patients were also receiving $\beta$ blockers. The results showed that the survival among patients receiving $\beta$ blockers $(83 \%, 66 \%$, and $52 \%$ at one, two, and three years) was significantly better than control group $(46 \%, 19 \%$, and $10 \%) . \beta$ Blockers were also associated with a survival benefit in patients with an ejection fraction $>30 \%$, and in those in whom the ratio of the apex cardiogram rapid filling wave to total height was $>15 \% .^{5}$

Fifteen of the patients who had received $\beta$ blockers for at least six months and who had improved were studied after withdrawal of the drug. ${ }^{6}$ Six patients had a profound deterioration of their condition, and the remaining patients had a significant decrease in ejection fraction with signs of compromised diastolic function seen as pathological apex curves and an increase in the third heart sound. These changes were reversed within several weeks to a few months after re-administration of $\beta$ blockade.

It was concluded from these studies that a pathological response to sympathetic stimulation was likely to be an aetiological factor for congestive cardiomyopathy. $\beta$ Blockers are able to modulate this defect, but the fact that symptoms re-emerge after stopping treatment suggests that $\beta$ blockers are not curative.

\section{Emerging findings}

In the 1980s Bristow et al showed that the adenylate cyclase response to $\beta$ adrenoceptor blockade was attenuated in patients with heart failure compared with controls. ${ }^{7}$ This was later found to be caused by a reduction in the steady-state abundance of mRNA for $\beta_{1}$ adrenoceptors in patients with heart failure. ${ }^{8}$ Anderson et al conducted a long term randomised trial of low dose metoprolol in 50 
patients but observed only a modest beneficial effect of $\beta$ blockade relative to control patients. ${ }^{9}$

In 1989 it was reported that the acute effect of $\beta$ blocker treatment was to produce a deterioration in cardiac index, and that it was only after at least three months of treatment that cardiac index normalised. $\beta$ Blockers were also shown to be devoid of any acute effect on end systolic pressure whereas in the longer term there was an improvement in systolic function. These findings highlighted the need for treatment to be administered at an adequate dose and for an appropriate duration.

An international multicentre study, the metoprolol in dilated cardiomyopathy (MDC) trial, was started in 1985 to evaluate the long term effect of metoprolol in patients with dilated cardiomyopathy. ${ }^{10}$ The primary end points were survival and need for heart transplantation, which was determined by a blinded committee and based on predetermined criteria for establishing deterioration in myocardial function. This second end point was considered to be important because the study included much younger patients (mean age 49 years) than those recruited into conventional heart failure trials.

The study was analysed when 383 patients had been followed for at least 12 months; there was a group of patients who had been followed for 18 months. The results showed a modest beneficial effect of metoprolol during the first year of treatment, shown by a $34 \%$ reduction in risk of reaching one of the two end points with metoprolol. However, separate analysis of the risk of heart transplantation showed a dramatic shift in favour of the treated group. The trial also demonstrated that metoprolol was well tolerated in the long term as shown by the very low withdrawal rate. The withdrawal rate during the first six weeks was similar to that in the placebo group but by the end of the first year there were far fewer withdrawals in the metoprolol group because of deterioration in cardiac function.

The cardiac insufficiency bisoprolol study (CIBIS), published in 1994, investigated the effect of bisoprolol in heart failure. ${ }^{11}$ The CIBIS trial was the first large scale study of $\beta$ blocker treatment in heart failure using mortality as the main end point; the MDC trial had a combined end point. A total of 642 patients with chronic heart failure of various causes, including idiopathic as well as ischaemic cardiomyopathy, and a left ventricular ejection fraction of $<40 \%$, were entered into this placebo controlled, randomised study.

Patients were in New York Heart Association (NYHA) functional class III (95\%) or IV $(5 \%)$. They were followed up for a mean of
1.9 years, and bisoprolol was well tolerated in patients with idiopathic as well as ischaemic cardiomyopathy. There was an overall $20 \%$ reduction in mortality for patients receiving bisoprolol although this decrease was non-significant $(\mathrm{p}=0.22)$. Bisoprolol did improve the functional status of the patients; fewer patients in the bisoprolol group required hospitalisation for cardiac decompensation (90 taking placebo $v 61$ taking bisoprolol, $\mathrm{p}<0.01$ ), and more patients improved at least one NYHA functional class (48 taking placebo $v 68$ taking bisoprolol, $\mathrm{p}=0.04)$. A retrospective subgroup analysis suggested that the reduction in mortality was greater for those with non-ischaemic cardiomyopathy $(\mathrm{p}=$ $0.01) .{ }^{11}$

\section{Conclusions}

Until 1985 the role of $\beta$ blockers in the management of heart failure had only been investigated in small, short term trials. More recently, the results of large, longer term multicentre evaluations have been analysed and reported. During the 23 years between 1973 and 1996 the consensus for using $\beta$ blockers to treat heart failure has changed from being unacceptable to plausible - in a few centres $\beta$ blockers have become accepted as standard treatment.

This experience reflects the difficulty of establishing a new treatment before elucidating a scientific rationale for its mechanism of action. Even now additional aspects are being discovered that will help to explain more fully the way in which $\beta$ blockers act in patients with congestive heart failure.

1 Waagstein F, Hjalmarson A, Varnauskas E, et al. Effect of chronic beta-adrenergic receptor blockade in congestive cardiomyopathy. Br Heart $\mathcal{f}$ 1975;37:1022-36.

2 Swedberg K Initial experience with beta-blockers in dilated cardiomyopathy. Am f Cardiol 1993;71:30-8C.

3 Swedberg K, Hjalmarson A, Waagstein F, et al. Beneficial effects of long-term $\beta$ blockade in congestive heart failure Br Heart $\mathcal{F} 1980 ; 44: 117-33$

4 Goodwin F, Oakley CM. The cardiomyopathies. Br Heart $\mathscr{f}$ 1972;34:545-52.

5 Swedberg K, Hjalmarson A, Waagstein F, et al. Prolongation of survival in congestive cardiomyopathy by betareceptor blockade. Lancet 1979 ;ii:1374-6.

6 Swedberg K, Hjalmarson A, Waagstein F, et al. Adverse effects of $\beta$ blockade withdrawal in patients with congestive cardiomyopathy. Br Heart 7 1980;44:134-42.

7 Bristow MR, Herschenberger RE, Fowler M, et al. $\beta 1$ - and $\beta 2$-adrenergic receptor subpopulations in normal and 2ailing human ventricular myocardium: coupling of both failing human ventricular myocardium: coupling of both receptor subtypes to muscle contraction and selective $\beta$ receptor down-regulation in heart failure. Circ Res 1986; 59.297-309.

8 Bristow MR, Minobe W, Raynolds MV, et al. Reduced $\beta 1$ receptor mRNA abundance in the failing human heart. $\mathscr{f}$ Clin Invest 1993;92:2737-45.

9 Anderson JL, Lutz JR, Gilbert EM, et al. A randomized trial of low dose beta blockade therapy for idiopathic dilated cardiomyopathy. Am f Cardiol 1985;55:471-5.

10 Waagstein F, Bristow MR, Swedberg K, et al. Beneficia effects of metoprolol in idiopathic dilated cardiomyopathy. Lancet 1993;342:1441-6.

11 CIBIS Investigators and Committees. A randomized trial of beta-blockade in heart failure. The cardiac insufficiency bisoprolol study (CIBIS). Circulation 1994;90: $1765-73$. 